\title{
Detection of Carbapenemase-Producing Enterobacteriaceae in the Baltic Countries and St. Petersburg Area
}

\author{
Anastasia Pavelkovich, ${ }^{1,2}$ Arta Balode, ${ }^{3}$ Petra Edquist, ${ }^{4}$ Svetlana Egorova, ${ }^{5}$ \\ Marina Ivanova, ${ }^{2}$ Lidia Kaftyreva, ${ }^{5}$ Irina Konovalenko, ${ }^{6}$ Siiri Kõljalg, ${ }^{1}$ Jana Lillo, ${ }^{1}$ \\ Lidia Lipskaya, ${ }^{7}$ Jolanta Miciuleviciene, ${ }^{8}$ Kristiine Pai, ${ }^{1}$ Kristel Parv, ${ }^{1}$ Katri Pärna, ${ }^{1}$ \\ Tiiu Rööp, ${ }^{1}$ Epp Sepp, ${ }^{1}$ Jelena Štšepetova, ${ }^{1}$ and Paul Naaber ${ }^{1,9}$ \\ ${ }^{1}$ Department of Microbiology, University of Tartu, Ravila 19, 50411 Tartu, Estonia \\ ${ }^{2}$ East-Tallinn Central Hospital, Ravi 18, 10138 Tallinn, Estonia \\ ${ }^{3}$ Rìga Stradiņš University, 16 Dzirciema Street, Rīga, LV-1007, Latvia \\ ${ }^{4}$ Smittskyddsinstitutet, Folkhälsomyndigheten, 17182 Solna, Sweden \\ ${ }^{5}$ Institut Pasteur in Saint Petersburg, Ul Mira 14, Saint Petersburg 197101, Russia \\ ${ }^{6}$ St. Petersburg Hospital No. 31, Pr. Dinamo 3, Saint Petersburg 197110, Russia \\ ${ }^{7}$ St. Petersburg Hospital No. 40, Ul Borisova 9, Sestroretsk, Saint Petersburg 197706, Russia \\ ${ }^{8}$ Vilnius City Clinical Hospital, Antakalnio Street 57, LT-10007 Vilnius, Lithuania \\ ${ }^{9}$ Quattromed HTI Laboratories, Väike-Paala 1, 11415 Tallinn, Estonia
}

Correspondence should be addressed to Paul Naaber; paul.naaber@quattromed.ee

Received 4 December 2013; Revised 18 January 2014; Accepted 21 January 2014; Published 4 March 2014

Academic Editor: Karmen Torkar

Copyright (C) 2014 Anastasia Pavelkovich et al. This is an open access article distributed under the Creative Commons Attribution License, which permits unrestricted use, distribution, and reproduction in any medium, provided the original work is properly cited.

\begin{abstract}
The spread of carbapenemase-producing Enterobacteriaceae is a global problem; however, no exact data on the epidemiology of carbapenemase in the Baltic countries and St. Petersburg area is available. We aimed to evaluate the epidemiology of carbapenemaseproducing Escherichia coli and Klebsiella pneumoniae in the Baltic States and St. Petersburg, Russia, and to compare the different methods for carbapenemase detection. From January to May 2012, all K. pneumoniae $(n=1983)$ and E. coli $(n=7774)$ clinical isolates from 20 institutions in Estonia, Latvia, Lithuania, and St. Petersburg, Russia were screened for carbapenem susceptibility. The IMP, VIM, GIM, NDM, KPC, and OXA-48 genes were detected using real-time PCR and the ability to hydrolyze ertapenem was determined using MALDI-TOF MS. Seventy-seven strains were found to be carbapenem nonsusceptible. From these, $15 \mathrm{~K}$. pneumoniae strains hydrolyzed ertapenem and carried the $b l a_{\mathrm{NDM}}$ gene. All of these strains carried integron 1 and most carried integron 3 as well as genes of the CTX-M-1 group. No carbapenemase-producing E. coli or K. pneumoniae strains were found in Estonia, Latvia, or Lithuania; however, NDM-positive K. pneumoniae was present in the hospital in St. Petersburg, Russia. A MALDI-TOF MS-based assay is a suitable and cost-effective method for the initial confirmation of carbapenemase production.
\end{abstract}

\section{Introduction}

Antibiotic resistance remains a major global public health problem that leads to increasing healthcare costs, extra length of hospital stay, and treatment failures [1]. The recent European Antimicrobial Resistance Surveillance Network (EARS-Net) report indicates that although the occurrence of antibiotic resistance in gram-positive pathogens appears to be stabilizing or even decreasing in some countries Europewide increase in antimicrobial resistance in gram-negative pathogens, such as Escherichia coli and Klebsiella pneumoniae, is occurring [1].

An emerging problem is the spread and increasing prevalence of carbapenemase-producing gram-negative bacteria. For example, outbreaks of Klebsiella pneumoniae carbapenemase (KPC)-positive K. pneumoniae occurred in the USA 
in 2001 and subsequently spread throughout the world; New Delhi metallo- $\beta$-lactamase (NDM-1)-positive K. pneumoniae was imported from India and spread to the United Kingdom in 2010 [2]. As high as a 50\% prevalence of metallo- $\beta$-lactamases were reported in $K$. pneumoniae blood isolates in Greece [3].

In a recent European study, most participants declared sporadic cases or outbreaks of carbapenemase-producing Enterobacteriaceae [4]. Although carbapenem nonsusceptible E. coli and $K$. pneumoniae cases have also been reported in the Baltic states, no exact epidemiological data are available, and these cases were not confirmed using molecular methods.

The detection of carbapenemases in clinical microbiology labs is challenging [5] because phenotypic tests are time consuming and difficult to interpret and the molecular methods are not available in routine diagnostic labs in our region. Additionally, the increasing number of new carbapenemases makes molecular tests unsuitable for the initial detection of carbapenemase production. Recently a MALDI-TOF MS (matrix-assisted laser desorption ionization-time of flight mass spectrometry) assay has been described as a potentially useful tool for the detection of $\beta$-lactamase activity $[6,7]$. This technique is based on the detection of $\beta$-lactams degradation products. However, this method has not yet been introduced in routine diagnostics or validated in different geographical regions.

The aim of our study was to evaluate the epidemiology of carbapenemases in the Baltic countries and St. Petersburg area by applying different mass-spectrometry and molecularbased detection methods.

\section{Materials and Methods}

2.1. Collection of Strains. A total of 20 institutions from the following Baltic countries and St. Petersburg area participated in our study: Estonia $(n=5)$, Lithuania $(n=3)$, Latvia $(n=4)$, and the Saint-Petersburg region in Russia $(n=8)$. From January 1 to May 31, 2012, all isolated E. coli and $K$. pneumoniae nonduplicated strains were screened for carbapenem nonsusceptibility according to European Committee of Antimicrobial Susceptibility Testing (EUCAST 2.0; applied in Baltic states) or Clinical and Laboratory Standard Institute (CLSI 2011; applied in Russia) standards in the participating labs. Carbapenem nonsusceptibility to ertapenem and/or meropenem was detected using disc-diffusion or broth dilution methods. Strains from all clinically relevant materials that were routinely sent to these microbiology labs were included. Environmental and surveillance samples were not included in our study. In total, 9757 strains were isolated and screened for carbapenem nonsusceptibility and included E. coli from hospitalized patients $(n=4799)$ and outpatients $(n=2975)$ and $K$. pneumoniae from hospitalized patients $(n=1631)$ and outpatients $(n=352)$. All screening positive strains were sent to and further investigated in the Estonian central lab.

2.2. Strains Data. Institution (numbers of beds, admissions, and patient days), strain-related (sampling date, origin of isolation/material, and resistance pattern detected in the local labs), and patient-related data (patient age, outpatient or hospitalized patient, and department type) were also collected for the carbapenem nonsusceptible strains.

2.3. Phenotypic Description of Strains. Species identification of these strains was confirmed in the central lab using MALDI-TOF MS (Maldi Biotyper, Bruker Daltonics GmbH, Germany). Carbapenem nonsusceptibility was confirmed using the agar-gradient method with ertapenem, meropenem, and imipenem strips (Liofilchem, Italy) on MullerHinton agar (Oxoid Limited, UK) according to the manufacturer's recommendations and was interpreted according to EUCAST standards (http://www.eucast.org/).

2.4. Mass-Spectrometry-Based Confirmation of Carbapenemase Production. Carbapenem nonsusceptible strains were analyzed using a mass-spectrometry-based semi quantitative resistance assay method that was described previously by Burckhardt and Sparbier $[8,9]$ and modified according to Dr. Katrin Sparbier's recommendations (personal communication).

Bacterial strains were grown for 18 to $24 \mathrm{~h}$ on $5 \%$ sheep blood agar plates (Microlabor, Estonia) at $36^{\circ} \mathrm{C}$. The cells were then resuspended in thirty microliters of $0.45 \% \mathrm{NaCl}$ solution with or without $1 \mathrm{~g} /$ litre ertapenem (MSD, USA) in $1.5-\mathrm{mL}$ Eppendorf tubes and the amount of bacteria filled a $1-\mu \mathrm{L}$ inoculation loop (corresponding to $3-6 \times 10^{8}$ cells, $1 \mathrm{~mL}$ of a McFarland 1-2). The suspension was incubated for $2 \mathrm{~h}$ at $37^{\circ} \mathrm{C}$ under agitation and then centrifuged for $2 \mathrm{~min}$ at $13.000 \mathrm{rpm}$ at $20^{\circ} \mathrm{C}$. One microliter of the cleared supernatant was added to each target spot and dried at room temperature, and $1 \mu \mathrm{L}$ of matrix (HCCA [ $\alpha$-cyano-4-hydroxycinnamic acid], high-pressure liquid chromatography [HPLC] grade; Fluka, Germany) was added to each target spot.

MALDI-TOF MS analysis was performed using a Microflex LT instrument (Bruker Daltonics GmbH, Germany) and 96-spot, polished-steel targets. The specifically modified protocol was used (0 to $1000 \mathrm{Da}, 12$ to $17 \%$ laser intensity). For one spectrum, approximately 200 to 300 shots were summed and the HCCA peak at $379 \mathrm{Da}$ was used for calibration. The mass spectrum between 438 and $530 \mathrm{Da}$ was analyzed using 3.0 software flexAnalysis (Bruker Daltonics $\mathrm{GmbH}$, Germany). Carbapenem nonsusceptible strains were analyzed in six reaction sets and each set contained positive (carbapenemase-producing strain) and negative controls (strain incapable of carbapenemase production), and during each reaction set, the characteristic mass spectrum of pure ertapenem was measured.

An automated evaluation algorithm for the MALDITOF MS-based functional $\beta$-lactamase assay (MSBL assay) was implemented using Bruker Daltonics software prototype (which is underdevelopment and not yet commercially available) that was employed in cooperation with Sparbier et al. [10]. This software automatically calculates the logarithm of the sum ratio of the intensity of all peaks that corresponded to the hydrolyzed form and the sum of the intensities of all peaks that corresponded to the non-hydrolyzed form of 
the antibiotic $(\log R Q)$. The results were displayed in a box plot diagram [10]. This automated evaluation was used as a control method for the visual analysis of the spectra.

2.5. Molecular Characterization of Strains. Total bacteria DNA from the clinical samples was extracted using the QIAamp DNA Mini Kit (Qiagen, Germany). Plasmid DNA was extracted using the NucleoSpin Plasmid Quick Pure kit (Macherey-Nagel, France).

Genes encoding IMP, VIM, GIM, NDM, OXA-48, and KPC-group carbapenemases were detected using a multiplex real-time PCR reaction that was based on the protocols published previously by Mendes et al. [11], Poirel et al. [12], Samuelsen et al. [13], and Chen et al. [14]. Amplification was performed in a $20-\mu \mathrm{L}$ mixture that contained $10 \mu \mathrm{L}$ of a Power SYBR Green PCR Master Mix (Applied Biosystems, UK) and six pairs of primers (concentration rate is $10 \mu \mathrm{M}$ ) in 3 different PCRs (Table 1(a)) using Applied Biosystems StepOnePlus Real-Time PCR system (Life Technologies, USA). The PCR conditions were as follows: 1 cycle at $50^{\circ} \mathrm{C}$ for $2 \mathrm{~min}$, initial denaturation at $94^{\circ} \mathrm{C}$ for $5 \mathrm{~min} ; 35$ cycles of $94^{\circ} \mathrm{C}$ for $20 \mathrm{~s}, 53^{\circ} \mathrm{C}$ for $45 \mathrm{~s}$ for the $b l a_{\mathrm{IMP}}, b l a_{\mathrm{VIM}}$, and $b l a_{\mathrm{GIM}}$ genes, or $60^{\circ} \mathrm{C}$ for $45 \mathrm{~s}$ for the $b l a_{\mathrm{OXA}-48}, b l a_{\mathrm{NDM}}$, and $b l a_{\mathrm{KPC}}$ genes, and $72^{\circ} \mathrm{C}$ for $30 \mathrm{~s}$; and a melt curve step at $95^{\circ} \mathrm{C}$ for $15 \mathrm{~s}, 60^{\circ} \mathrm{C}$ for $1 \mathrm{~min}$, and $95^{\circ} \mathrm{C}$ for $15 \mathrm{~s}$.

The presence of different $b l a_{\mathrm{CTX} \text {-M }}$ subgroups was determined using a multiplex real-time PCR protocol [15] that displayed the group designation (CTX-M-1/-2/-9 and 8/25) of the $b l a_{\text {СТХ-м}}$-positive isolates. The method used one primer pair that was specific for all four groups. Four TaqMan probes were included in the reactions and bound to PCR products according to the CTX-M subgroup.

To detect integrons, real-time PCR was performed as previously described [16] with a reaction mixture of $25 \mu \mathrm{L}$ that contained $5 \mu \mathrm{L}$ of DNA template, $12.5 \mu \mathrm{L}$ of TaqMan universal master mix (Applied Biosystem, USA), $6 \mathrm{mM} \mathrm{MgCl}_{2}, 0.4 \mu \mathrm{M}$ of each primer (int1-(LC1-LC5), int2-(LC2-LC3), and int3(LC1-LC2)), and $0.2 \mu \mathrm{M}$ of each probe (int1, int2, and int3; Table $1(\mathrm{~b})$ ). The programme was as follows: $10 \mathrm{~min}$ initial step at $95^{\circ} \mathrm{C}$, followed by 45 cycles at $95^{\circ} \mathrm{C}$ for $30 \mathrm{sec}$ and $60^{\circ} \mathrm{C}$ for $1 \mathrm{~min}$, and it was performed using a 7500 real-time PCR detection system (Applied-Biosystem, USA) and optical grade, 96 -well plates. Assays were performed in triplicate, and a negative control was included on each plate.

2.6. Quality Control Strains. In the carbapenemases detection studies (MALDI-TOF based assay and real-time PCR) the following control strains were used: Swedish Institute for Communicable Disease Control (Sweden) carbapenemases detection control set including IMP-positive Pseudomonas aeruginosa Ps540, VIM-positive K. pneumoniae CCUG58547, GIM-positive $P$. aeruginosa, NDM-1-positive $K$. pneumoniae K275, OXA-48-positive K. pneumoniae Oxa241, KPCpositive K. pneumoniae K271, and MBL-positive Klebsiella pneumoniae KSKS2823 and EuSCAPE (European survey on carbapenemase-producing Enterobacteriaceae) project quality control set (provided by NEQAS) including KPCpositive K. pneumoniae 1940, KPC-2-positive K. pneumoniae

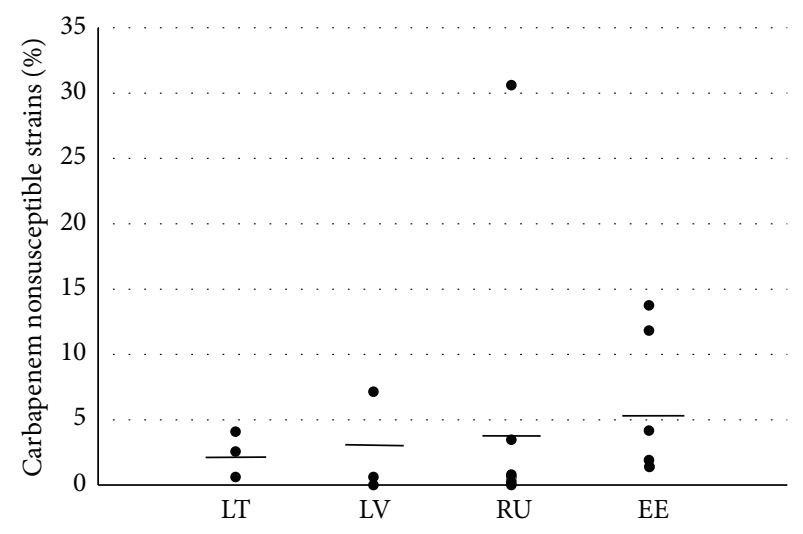

Figure 1: Percentages of carbapenem nonsusceptible Klebsiella pneumoniae strains in particular hospitals (dots) and the country averages (lines). LT: Lithuania, LV: Latvia, RU: Russia, St. Petersburg, and EE: Estonia.

1944, KPC-3-positive K. pneumoniae 1942, OXA-48-positive K. pneumoniae 1943, VIM-1-positive K. pneumoniae 1945, NDM-1-positive K. pneumoniae strains 1946 and 1948, IMP-1positive K. pneumoniae 1947, and NDM-1-positive Enterobacter aerogenes 1941.

In CTX-M detection studies we used E. coli CCUG55971 (CTX-M-1 group), E. coli CCUG55972 (CTX-M-2), E. coli CCUG55970 (CTX-M-9), and E. aerogenes isolate Rio3 (CTX-M-8/25) as positive controls. These strains were provided from Swedish Institute for Communicable Disease Control.

For integron detections the following strains were used as positive templates: E. coli/pBad::int1; E. coli/pGEMTeasy:: int2; and E. coli/pBad::int3 provided from the Department of Bacteriology of University of Limoges (France).

For negative controls wild-type E. coli and K. pneumoniae clinical strains were used.

\section{Results}

According to initial screening and identification confirmation, 80 isolates appeared to be nonsusceptible to carbapenem. After minimal inhibitory concentration (MIC) confirmation using the agar-gradient method, 77 strains had MIC values above the epidemiological cut-off according to the EUCAST standard for any tested carbapenem (nonwild type strains), including $73 \mathrm{~K}$. pneumoniae and 4 E. coli strains. The prevalence of the carbapenem nonsusceptible strains in the institutions of the participating countries is shown in Figure 1.

3.1. Carbapenemase Production Confirmation by MALDI-TOF MS. Of the 77 phenotypically carbapenem nonsusceptible strains, 15 were able to hydrolyze ertapenem, as detected by the MSBL assay. The spectra of all carbapenemase-producing strains showed the hydrolyzed decarboxylated product of ertapenem $(450.5 \mathrm{Da})$, which corresponded to high-strength carbapenemase (Figure 2). The visual- and software-based evaluations of the results gave the same results. An example of 
TABle 1: (a) Primers for the detection of carbapenemase-encoding genes. (b) Primers and probes used for multiplex real-time PCR detection of classes 1, 2, and 3 integrons.

(a)

\begin{tabular}{lccc}
\hline Reaction number & Primers & Oligonucleotide sequence $\left(5^{\prime}-3^{\prime}\right)$ & Volume for reaction \\
\hline & IMP (forward) & GAATAGRRTGGCTTAAYTCTC & $2.5 \mu \mathrm{L}$ \\
PCR & IMP (reverse) & CCAAACYACTASGTTATC & $2.5 \mu \mathrm{L}$ \\
Mix 1 & VIM (forward) & ATTTGGTCGCATATCGCAAC & $0.25 \mu \mathrm{L}$ \\
& VIM (reverse) & TCAATTAGCTCTTGGGCTGAC & $0.25 \mu \mathrm{L}$ \\
& GIM (forward) & CGGAACGACCATTTGAATGG & $0.25 \mu \mathrm{L}$ \\
GIM (reverse) & CAATATTATGCACCCGGTCG & $0.25 \mu \mathrm{L}$ \\
PCR & NDM (forward) & ATCATGCTGGCCTTGGGGAA & $0.5 \mu \mathrm{L}$ \\
& OXA 48 (forward) & GCGTGGTTAAGGATGAACAC & $0.5 \mu \mathrm{L}$ \\
PCR 2 & OXA 48 (reverse) & CATCAAGTTCAACCCAACCG & $0.5 \mu \mathrm{L}$ \\
Mix 3 & KPC (forward) & GGCAGTCGGAGACAAAACC & $0.5 \mu \mathrm{L}$ \\
\hline
\end{tabular}

(b)

\begin{tabular}{lcc}
\hline Primers/probes & Oligonucleotide sequence $\left(5^{\prime}-3^{\prime}\right)$ & Location \\
\hline intI1-LC1 & GCCTTGATGTTACCCGAGAG \\
intI1-LC5 & GATCGGTCGAATGCGTGT \\
intI2-LC2 & TGCTTTTCCCACCCTTACC \\
intI2-LC3 & GACGGCTACCCTCTGTTATCTC \\
intI3-LC1 & GCCACCACTTGTTTGAGGA \\
intI3-LC2 & GGATGTCTGTGCCTGCTTG \\
\hline intI1-probe & ATTCCTGGCCGTGGTTCTGGGTTTT & intI2 \\
intI2-probe & TGGATACTCGCAAACAAGTTATTTTTACGCTG \\
intI3-probe & CGCCACTCATTCGCCACCCA & intI1 \\
\hline
\end{tabular}

the ertapenem hydrolyzation spectra visualised with software prototype is presented in Figure 3.

3.2. Molecular Detection of Carbapenemase-Encoding Genes. When analysing these 77 carbapenem nonsusceptible isolates for carbapenemase-encoding genes, we found 15 isolates that were positive for the bla $a_{\mathrm{NDM}}$ gene. All of the other 62 isolates were negative for the $b l a_{\mathrm{IMP}}, b l a_{\mathrm{VIM}}, b l a_{\mathrm{GIM}}, b l a_{\mathrm{OXA}-48}$, $b l a_{\mathrm{NDM}}$, or $b l a_{\mathrm{KPC}}$ genes. The 15 isolates with the NDM gene were also found to hydrolyze ertapenem by the MSBL assay.

3.3. Characterization of Strains. All carbapenemase producers were identified as K. pneumoniae and were isolated from one St. Petersburg (Russia) hospital (Table 2). Most of these strains were isolated from intensive care patients and were isolated from different clinical materials. All of these strains carried integron 1, and most carried integron 3 and the CTXM-1 group of genes. All strains were resistant to all of the tested carbapenems, fluoroquinolones, and aminoglycosides. When comparing the minimal inhibitory concentration ranges of the carbapenemase producers and nonproducers, we found that the producers had imipenem MICs $\geq 8 \mu \mathrm{g} / \mathrm{mL}$ and nonproducers had MICs $\leq 4 \mu \mathrm{g} / \mathrm{mL}$. For the other carbapenems the ranges overlapped.

\section{Discussion}

This was the first study to investigate carbapenem resistance in the Baltic countries and St. Petersburg area that included patients and sample types from the multiple largest medical institutions in Estonia, Latvia, Lithuania, and St. Petersburg and to apply molecular methods for characterization of strains. We found high variation in carbapenem nonsusceptibility (MICs above the epidemiological cut-off values) between different institutions within particular countries (highest in Russia: from $0 \%$ to $30 \%$ ). However, variations between the country averages were not so remarkable. Although we found carbapenem nonsusceptible strains in the Baltic states (Estonia, Latvia, and Lithuania), as was described in previous EARS-Net reports, none of these were actually carbapenemase producers. Therefore, after screening nearly 10000 E. coli and K. pneumoniae strains, we conclude that carbapenemases are not yet a problem in Baltic countries. In these carbapenem nonsusceptible strains, some other mechanisms, for example, production of CTX-M as well as acquired cephalosporinases along with porin loss or changes in efflux, are suspected. In these cases, carbapenem resistance was usually low (relatively low MICs) and thus infections could be treated with higher doses of carbapenems. These strains appear to exhibit low spreading potential. 


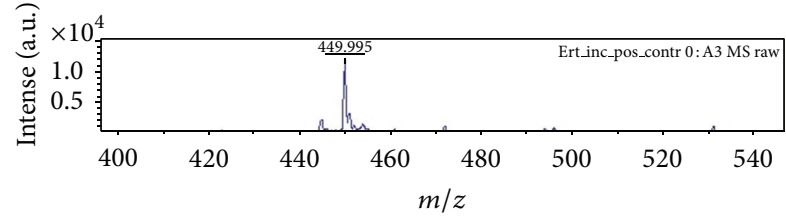

(a)

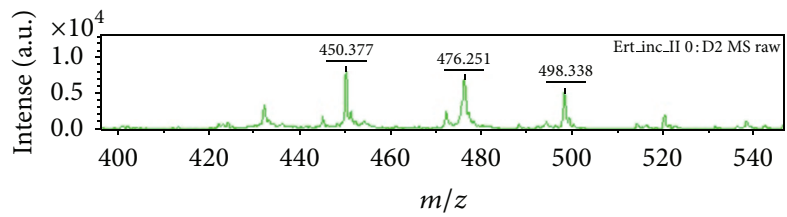

(b)

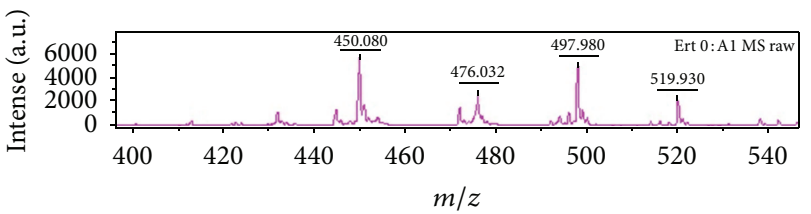

(c)

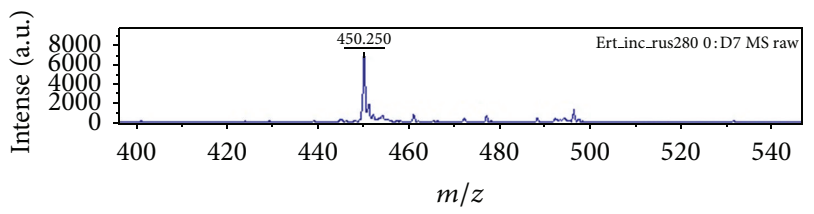

(d)

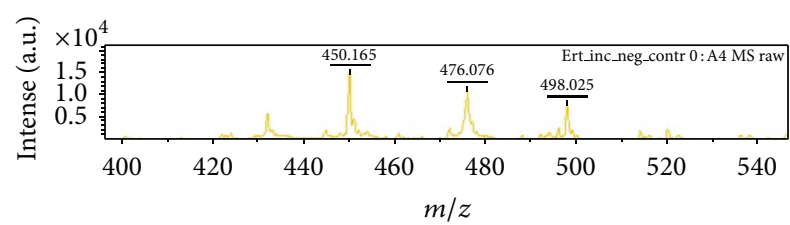

(e)

Figure 2: MALDI-TOF MS spectrum showing ertapenem and its degradation products. Spectrum related to (a) positive control (Klebsiella pneumoniae metallo- $\beta$-lactamase positive): hydrolyzed decarboxylated product of ertapenem (450.5 Da); (b) incubated ertapenem solution: hydrolyzed decarboxylated product of ertapenem $(450.5 \mathrm{Da})$, ertapenem molecule $(476.5 \mathrm{Da})$, and ertapenem sodium adduct (498.5 Da); (c) pure ertapenem solution: hydrolyzed decarboxylated product of ertapenem $(450.5 \mathrm{Da})$, ertapenem molecule $(476.5 \mathrm{Da})$, ertapenem sodium adduct (498.5 Da), and ertapenem disodium adduct (520.5 Da); (d) New Delhi Metallo- $\beta$-lactamase-positive strain (Klebsiella pneumoniae): hydrolyzed decarboxylated product of ertapenem (450.5 Da); (e) negative control (noncarbapenemase-producing Klebsiella pneumoniae): hydrolyzed decarboxylated product of ertapenem (450.5 Da), ertapenem molecule $(476.5 \mathrm{Da})$, and ertapenem sodium adduct $(498.5 \mathrm{Da})$.

On the contrary, carbapenemase producers are associated with therapy failures, outbreaks, and increased mortality [17]. However, our study revealed high prevalence of NDMtype carbapenemase-producing $K$. pneumoniae from one St. Petersburg hospital. Infection control and antimicrobial

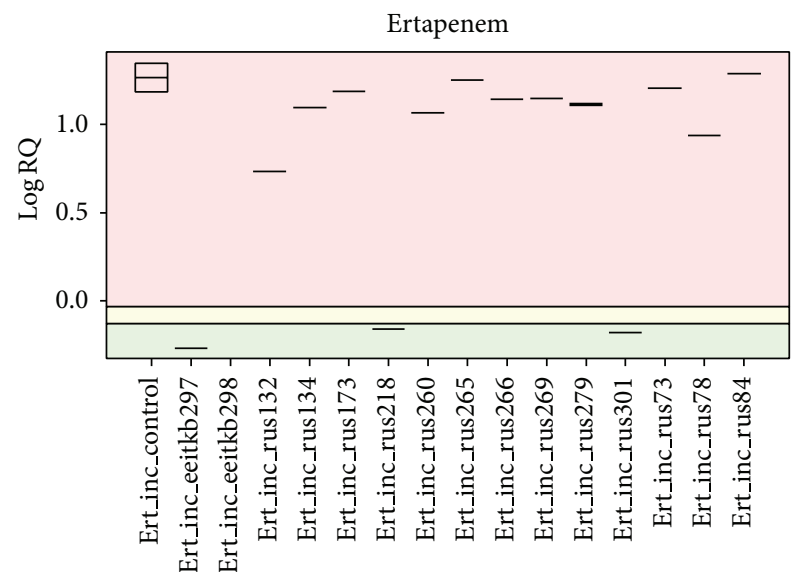

FIgURE 3: Evaluation of the MALDI-TOF MS spectra of 16 different Klebsiella pneumoniae strains using the new software prototype. Differences in the hydrolysis rates of the different strains can easily be detected: green area: no hydrolysis; yellow area: intermediate hydrolysis; red area: hydrolysis.

resistance surveillance institutions of neighboring Baltic States should be alerted when spreading of this strain occurs.

In our study, we also evaluated different methods, such as MALDI-TOF, MICs of different carbapenems, and realtime PCR, for the detection of carbapenemases in E. coli and $K$. pneumoniae isolates. In this set of strains, we found good correlation between functional assay, such as the MALDITOF assay and molecular detections of specific carbapenemase genes. Thus, from a sensitivity and specificity point of view, we found these methods to be equal. However, all of these methods have some advantages and disadvantages, as discussed below. The limitation of our study for evaluating the different methods was the epidemiological situation in the studied region, where only one type (NDM) of carbapenemase was present.

Whereas the molecular methods and the usage of carbapenems and the combinations of carbapenems and carbapenemase inhibitors have been available for years for carbapenemase detection, the MALDI-TOF assay is a novel method that is not fully standardized and has not been tested for different strains sets and epidemiological situations. However, carbapenem resistance detection using MALDI-TOF MS has many advantages when compared to synergy/inhibition-based phenotypic methods or PCR. First, depending on the type of carbapenemase, the results can be available as soon as $2-3 \mathrm{~h}$ after the start of incubation. This is especially useful in an outbreak situation when the carbapenemase has already been identified. The principle of degradation-product monitoring is universal for the detection of other enzymatic resistance mechanisms, such as extended spectrum $\beta$-lactamases (ESBL). Second, this method is comparatively easy to perform [8]. Third, the cost per determination is relatively low and is less than one EUR per reaction [8]. The only problem may be the cost of the equipment. However, MALDI-TOF MS has become increasingly used in routine diagnostic labs over recent years for the identification of pathogens. 
TABLE 2: Comparison of carbapenem nonwild type Klebsiella pneumoniae strains with and without carbapenemase production.

\begin{tabular}{|c|c|c|c|}
\hline & & $\begin{array}{c}\text { Carbapenemase } \\
\text { producers } \\
(n=15)\end{array}$ & $\begin{array}{c}\text { Carbapenemase } \\
\text { nonproducers } \\
(n=58) \\
\end{array}$ \\
\hline \multirow{4}{*}{ Country } & Russia & 15 & 7 \\
\hline & Estonia & 0 & 37 \\
\hline & Latvia & 0 & 8 \\
\hline & Lithuania & 0 & 6 \\
\hline \multirow{4}{*}{ Department } & Intensive care & 14 & 8 \\
\hline & Surgical & 0 & 16 \\
\hline & Medical & 1 & 31 \\
\hline & Outpatients & 0 & 3 \\
\hline \multirow{5}{*}{ Material } & Blood & 1 & 1 \\
\hline & Pus/wound & 2 & 11 \\
\hline & Urine & 5 & 39 \\
\hline & Lower respiratory & 7 & 6 \\
\hline & Upper respiratory & 0 & 1 \\
\hline \multirow{8}{*}{$\begin{array}{l}\text { Genes detected (number of } \\
\text { positive strains) }\end{array}$} & NDM & 15 & 0 \\
\hline & IMP, VIM, GIM, KPC or OXA48 & 0 & 0 \\
\hline & CTX-M-1 group & 13 & 50 \\
\hline & CTX-M-2 group & 0 & 2 \\
\hline & CTX-M-8 or 9 & 0 & 0 \\
\hline & Integron 1 & 15 & 52 \\
\hline & Integron 2 & 0 & 1 \\
\hline & Integron 3 & $11^{1}$ & $25^{1}$ \\
\hline \multirow{3}{*}{$\begin{array}{l}\text { MIC values, mg/L } \\
\text { (range/median) }\end{array}$} & Ertapenem & $\geq 32^{2}$ & $0.125-\geq 32 / 6^{2}$ \\
\hline & Meropenem & $4-\geq 32 / 12^{3}$ & $0.032-4 / 0.5^{3}$ \\
\hline & Imipenem & $8-\geq 32 / 32^{4}$ & $0.094-4 / 0.5^{4}$ \\
\hline \multirow{2}{*}{$\begin{array}{l}\text { Resistance (numbers: } \\
R+I / \text { tested strains) }\end{array}$} & Ciprofloxacin & $15 / 15$ & $49 / 51$ \\
\hline & Gentamycin & $15 / 15^{5}$ & $34 / 49^{5}$ \\
\hline
\end{tabular}

Unfortunately, a semiquantitative assay does not allow for specification of the enzyme. Currently, a software tool supporting an automated evaluation is under development which will be able to calculate the hydrolysis capacity (Bruker Daltonics GmbH, Germany) [18]. A prototype of this software was also used in our study to compare it with the manual method, and it was easy to use and gave reliable results with our strain set.

PCR is the fastest way to determine which family of $\beta$-lactamase is present. However, PCR techniques are not used in the majority of routine laboratories because of high costs, absence of real-time equipment, and qualified personnel. In addition, this method can detect only previously described enzymes. Mutations in a carbapenemase-encoding gene could lead to negative results.

We found that of the carbapenem nonsusceptible strains, most carbapenemase producers and nonproducers carried the CTX-M 1 group of ESBL genes and class 1 integrons. Although the class 3 integron was also found in carbapenemase nonproducers, it was more common in NDM-positive K. pneumoniae strains. In previous studies, a high prevalence of class 1 integrons in $K$. pneumoniae and its relation with NDM genes have been reported [19-21]. However, relations between carbapenem nonsusceptibility (carbapenemase related or nonrelated) and the presence of class 3 integrons have not yet been described and require further investigation.

In conclusion, carbapenemase-producing E. coli or $K$. pneumoniae were not found in Baltic countries, but NDMpositive K. pneumoniae was present in St. Petersburg. In our current epidemiological and economic situation, the MALDI-TOF assay seems to be suitable and cost-effective method for the initial confirmation of carbapenemase production.

\section{Conflict of Interests}

The authors declare that there is no conflict of interests regarding the publication of this paper. 


\section{Acknowledgments}

The authors are grateful to Ruta Ambrazaitiene, Gintaras Makstutis, Dace Rudzite, Tatjana Djundika, Nataliya Vedernikova, Olga Morozova, Tatyana Kurchikova, Maria Paysetchkaya, Marina Smirnova, Svetlana Rudenko, Irina Zolotuhhina, Kaisa Kirs, Krista Lõivukene, Natalja Kamonina, and Anna Tisler for their help in strain collection. They would also like to thank Dr. Katrin Sparbier and Christoph Lange (Bruker Daltonics GmbH, Germany) for assistance in the performance and interpretation of the MALDI-TOF MS assay; Dr. O. Barraud (Department of Bacteriology, Medical Faculty, University of Limoges, France), who kindly provided integron-positive control strains; the Swedish Institute for Communicable Disease Control for the set of carbapenemase and ESBL-positive control strains and methodology. This study was supported by grants of the Baltic Antibiotic Resistance collaborative Network (BARN), European Union through the European Regional Development Fund (ARMMD Project no. 3.2.0701.11-0013), Estonian Ministry of Education and Research (target financing no. SF0180132s08), and Estonian Science Foundation (Grant no. 9059).

\section{References}

[1] "Multidrug antibiotic resistance increasing in Europe," ECDC, 2012, http://www.ecdc.europa.eu/en/press/news/_layouts/forms/ News_DispForm.aspx? $\mathrm{ID}=563 \mathrm{\& List}=8 \mathrm{db} 7286 \mathrm{c}-\mathrm{fe} 2 \mathrm{~d}-476 \mathrm{c}-9133$ $18 \mathrm{ff} 4 \mathrm{cblb5} 68$.

[2] A. Marra, "NDM-1: a local clone emerges with worldwide aspirations," Future Microbiology, vol. 6, no. 2, pp. 137-141, 2011.

[3] EARS-Net Annual Report, 2011, http://www.ecdc.europa.eu/ en/publications/publications/antimicrobial-resistance-surveillance-europe-2011.pdf.

[4] C. Glasner, B. Albiger, G. Buist et al., "Carbapenemase-producing enterobacteriaceae in Europe: a survey among national experts from 39 countries, February 2013," Eurosurveillance, vol. 18, no. 28, article 3, 2013.

[5] P. Nordmann, M. Gniadkowski, C. G. Giske et al., "Identification and screening of carbapenemase-producing Enterobacteriaceae," Clinical Microbiology and Infection, vol. 18, no. 5, pp. 432-438, 2012.

[6] J. Hrabák, V. Študentová, R. Walková et al., "Detection of NDM1, VIM-1, KPC, OXA-48, and OXA-162 carbapenemases by MALDI-TOF mass spectrometry," Journal of Clinical Microbiology, vol. 50, no. 7, pp. 2441-2443, 2012.

[7] L. Wang, C. Han, W. Sui, M. Wang, and X. Lu, "MALDI-TOF MS applied to indirect carbapenemase detection: a validated procedure to clearly distinguish between carbapenemase-positive and carbapenemase-negative bacterial strains," Analytical and Bioanalytical Chemistry, vol. 405, no. 15, pp. 5259-5266, 2013.

[8] I. Burckhardt and S. Zimmermann, "Using matrix-assisted laser desorption ionization-time of flight mass spectrometry to detect carbapenem resistance within 1 to 2.5 hours," Journal of Clinical Microbiology, vol. 49, no. 9, pp. 3321-3324, 2011.

[9] K. Sparbier, S. Schubert, U. Weller, C. Boogen, and M. Kostrzewa, "Matrix-assisted laser desorption ionization-time of flight mass spectrometry-based functional assay for rapid detection of resistance against $\beta$-lactam antibiotics," Journal of Clinical Microbiology, vol. 50, no. 3, pp. 927-937, 2012.
[10] K. Sparbier, K. Lange, J. Jung, S. Schubert, and M. Kostrzewa, "An automated evaluation algorithm for then MALDI-TOF MS based functional $\beta$-lactamase assay," ASMS, A101, 2013, http://www.bruker.com/ru/products/mass-spectrometry-andseparations/literature/literature-room.html?eID=dam_frontend _push\&stream $=1 \&$ docID $=55242$.

[11] R. E. Mendes, K. A. Kiyota, J. Monteiro et al., "Rapid detection and identification of metallo- $\beta$-lactamase-encoding genes by multiplex real-time PCR assay and melt curve analysis," Journal of Clinical Microbiology, vol. 45, no. 2, pp. 544-547, 2007.

[12] L. Poirel, C. Héritier, V. Tolün, and P. Nordmann, "Emergence of oxacillinase-mediated resistance to imipenem in Klebsiella pneumoniae," Antimicrobial Agents and Chemotherapy, vol. 48, no. 1, pp. 15-22, 2004.

[13] Ø. Samuelsen, C. M. Thilesen, L. Heggelund, A. N. Vada, A. Kümmel, and A. Sundsfjord, "Identification of NDM-1-producing Enterobacteriaceae in Norway," Journal of Antimicrobial Chemotherapy, vol. 66, no. 3, pp. 670-672, 2011.

[14] L. Chen, K. D. Chavda, J. R. Mediavilla et al., "Multiplex realtime PCR for detection of an epidemic KPC-producing Klebsiella pneumoniae ST258 clone," Antimicrobial Agents and Chemotherapy, vol. 56, no. 6, pp. 3444-3447, 2012.

[15] C. I. Birkett, H. A. Ludlam, N. Woodford et al., "Real-time TaqMan PCR for rapid detection and typing of genes encoding CTX-M extended-spectrum $\beta$-lactamases," Journal of Medical Microbiology, vol. 56, part 1, pp. 52-55, 2007.

[16] O. Barraud, M. C. Baclet, F. Denis, and M. C. Ploy, "Quantitative multiplex real-time PCR for detecting class 1, 2 and 3 integrons," Journal of Antimicrobial Chemotherapy, vol. 65, no. 8, Article ID dkq167, pp. 1642-1645, 2010.

[17] P. Nordmann, T. Naas, and L. Poirel, "Global spread of carbapenemase producing Enterobacteriaceae," Emerging Infectious Diseases, vol. 17, no. 10, pp. 1791-1798, 2011.

[18] M. Kostrzewa and D. Orth, "Bruker Daltonik GmbH: MALDI TOF based microbial identification for the 21st century... and beyond," in Proceedings of the 23rd European Congress of Clinical Microbiology and Infectious Diseases, April 2013.

[19] R. Farzana, S. Shamsuzzaman, and K. Z. Mamun, "Isolation and molecular characterization of New Delhi metallo- $\beta$-lactamase1 producing superbug in Bangladesh," Journal of Infection in Developing Countries, vol. 7, no. 3, pp. 161-168, 2013.

[20] D. Yong, M. A. Toleman, C. G. Giske et al., "Characterization of a new metallo- $\beta$-lactamase gene, bla NDM-1, and a novel erythromycin esterase gene carried on a unique genetic structure in Klebsiella pneumoniae sequence type 14 from India," Antimicrobial Agents and Chemotherapy, vol. 53, no. 12, pp. 5046-5054, 2009.

[21] S. Derakhshan, S. N. Peerayeh, F. Fallah, B. Bakhshi, M. Rahbar, and A. Ashrafi, "Detection of class 1, 2, and 3 integrons among Klebsiella pneumoniae isolated from children in Tehran hospitals," Pediatric Infectious Diseases, vol. 1, no. 4, pp. 164-168, 2013. 

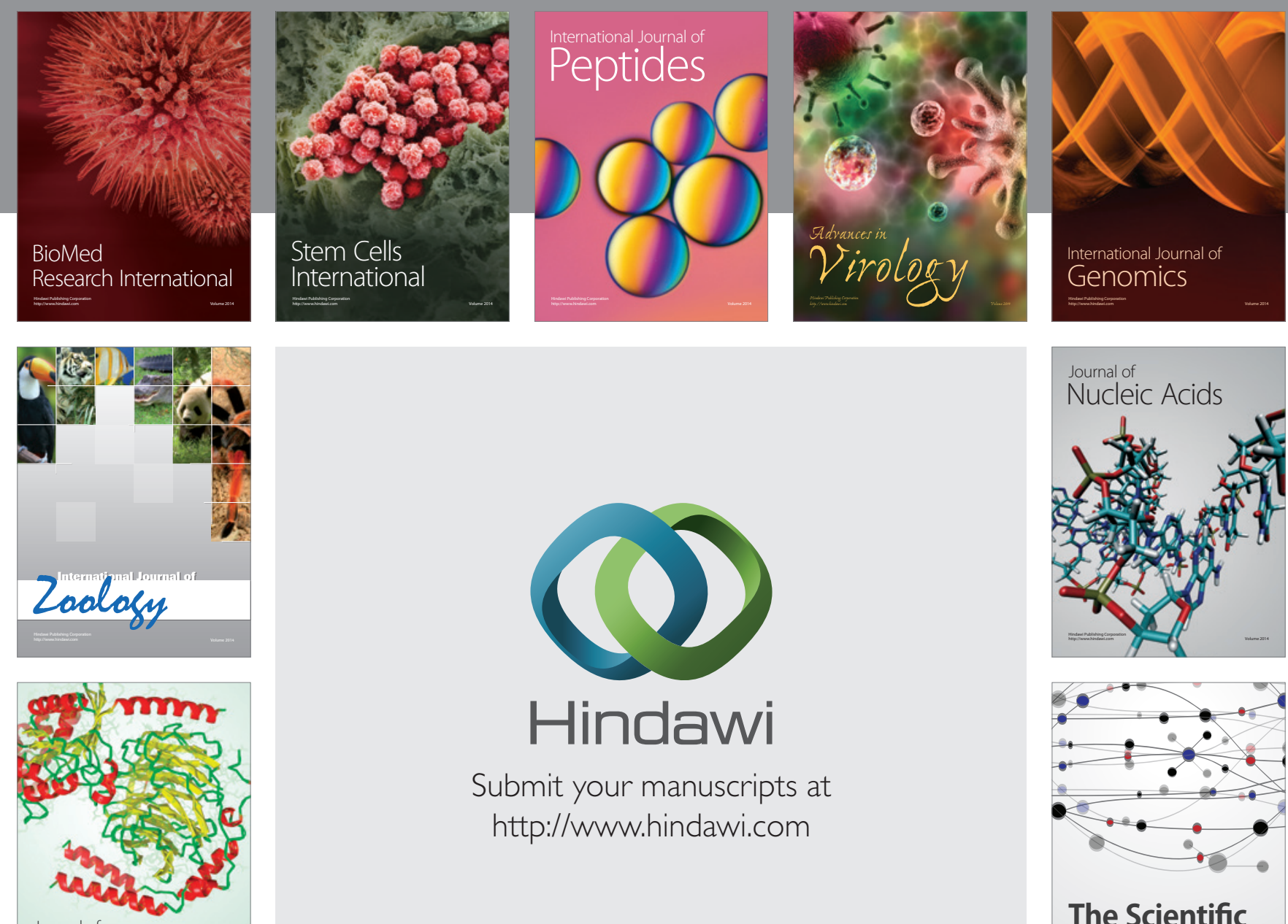

Submit your manuscripts at

http://www.hindawi.com

Journal of
Signal Transduction
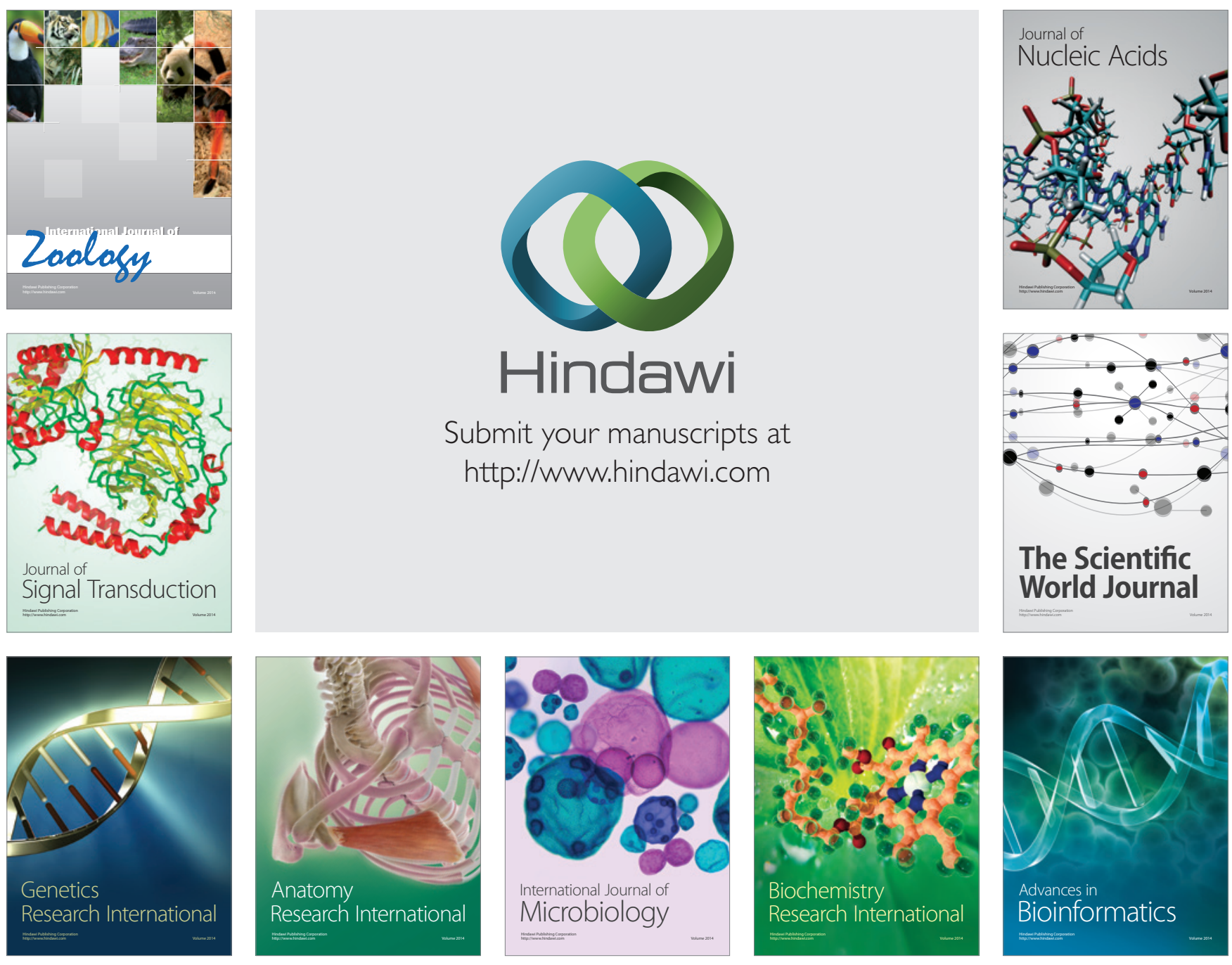

The Scientific World Journal
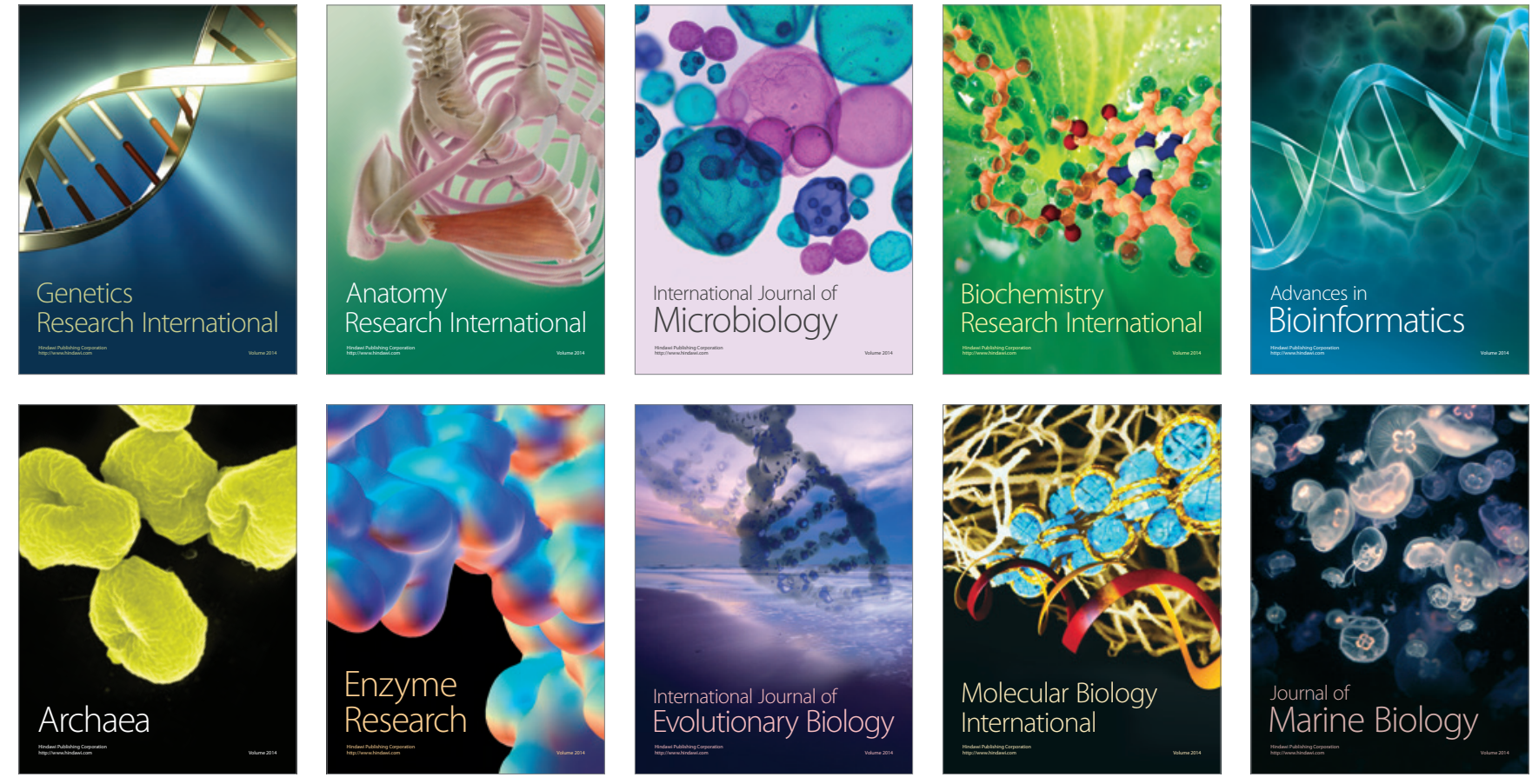